\title{
HYPERBOLICITY AND CYCLES
}

\author{
BY
}

\section{J. E. FRANKE AND J. F. SELGRADE}

\begin{abstract}
In this paper cycle points are defined without the assumption of Axiom $A$. The closure of the set of cycle points $C$ being quasi-hyperbolic is shown to be equivalent to Axiom A plus no cycles. Also we give a sufficient condition for $\mathcal{C}$ to equal the chain recurrent set. In proving these theorems, a spectral decomposition for quasi-hyperbolic invariant sets is used.
\end{abstract}

1. Introduction. Axiom A flows on compact connected manifolds have been widely studied since 1967 when S. Smale introduced the concept [11]. He has shown that the nonwandering set $\Omega$ of such a flow has a finite number of components each containing a transitive orbit. With the additional assumption of no cycles, $\Omega$ is stable under $C^{1}$-perturbations.

This work generalizes the $\Omega$-decomposition theorem and illustrates its essential features. Also, we establish a property which is equivalent to Axiom A plus no cycles and which may be more easily verified.

Theorem A. Spectral Decomposition Theorem. Let $f$ be $a C^{1}$ flow on a compact connected manifold $M$. If $\Lambda \subset M$ is a quasi-hyperbolic closed invariant set and contains the set of periodic orbits, then $\Lambda$ has a finite number of components. If, in addition, $\Lambda$ is chain recurrent then the periodic orbits are dense in $\Lambda$ and each component contains a transitive orbit.

Theorem B. Axiom A plus no cycles is equivalent to the existence of a quasi-hyperbolic invariant subset which contains all cycle points.

To prove Theorem B we study the relationship between the chain recurrent set $R$ and the closure of the set of cycle points $\mathcal{C}$, which we can define without assuming Axiom A. The principal result in this direction is

THEOREM C. Let $f$ be a $C^{1}$ flow on a compact connected manifold $M$. If the closure of the union of all minimal sets is quasi-hyperbolic then $\Re=C$.

2. Background. Let $f$ be a $C^{1}$ flow on a compact connected manifold $M$. A closed invariant set $\Lambda \subset M$ is hyperbolic if the tangent flow $T f$ leaves invariant a continuous splitting

Received by the editors March 25, 1977 and, in revised form, July 19, 1977.

AMS (MOS) subject classifications (1970). Primary 58F99; Secondary 58F15.

Key words and phrases. Flows, Axiom A, spectral decomposition, cycle points, chain recurrent, quasi-hyperbolic invariant set.

() American Mathematical Society 1979 


$$
T_{\Lambda} M=E^{s} \oplus E^{u} \oplus E
$$

where, for some $0<\lambda<1$ and some adapted Riemannian metric:

(i) if $v \in E^{u}$ and $t>0$ then $\left|T f_{t}(v)\right|>\lambda^{-t}|v|$;

(ii) if $v \in E^{s}$ and $t>0$ then $\left|T f_{t}(v)\right|<\lambda^{t}|v|$;

(iii) $E$ is the span of the vector field.

A weaker and more geometric condition than hyperbolicity is the following: a closed invariant set $\Lambda$ is quasi-hyperbolic if

(i) the span $E$ of the vector field of $\left.f\right|_{\Lambda}$ is a subbundle of $T_{\Lambda} M$;

(ii) the flow on $T_{\Lambda} M / E$ induced by the tangent flow has no nonzero bounded orbits.

A point $x$ is nonwandering if for each neighborhood $U$ of $x$ there is a $t>1$ such that $U \cap f_{t}(U) \neq \varnothing$. Let $\Omega$ denote the set of nonwandering points and $P$ the set of periodic points. The flow satisfies Axiom $A$ if $\Omega$ is hyperbolic and $\Omega=\mathrm{Cl} P$.

For subsets $\Lambda$ of $M$ and $J$ of $\mathbf{R}$, define $\Lambda \cdot J=f(\Lambda \times J)$. The $\alpha$ - and $\omega$-limit sets are defined by

$$
\alpha(x)=\bigcap_{t<0} \mathrm{Cl}(x \cdot(-\infty, t]), \quad \omega(x)=\bigcap_{t>0} \mathrm{Cl}(x \cdot[t, \infty)) .
$$

The nonwandering set of an Axiom A flow has finitely many components and each component is the $\alpha$ - and the $\omega$-limit set of one of its points [10], [11]. The Stable Manifold Theorem applied to one of these components $\Omega_{i}$ gives two submanifolds $W^{s}\left(\Omega_{i}\right)$ and $W^{u}\left(\Omega_{i}\right)$ which consist of the points which limit on $\Omega_{i}$ in positive and negative time, respectively. A cycle is a sequence of components $\Omega_{0}, \ldots, \Omega_{n}, n \geqslant 2$, with $\Omega_{0}=\Omega_{n}$ and $\Omega_{j} \neq \Omega_{k}$ otherwise, and

$$
W^{u}\left(\Omega_{i}\right) \cap W^{s}\left(\Omega_{i+1}\right) \neq \varnothing \quad \forall 0 \leqslant i \leqslant n-1 .
$$

Another way to generalize periodic motion is with chain recurrence [2], [5]. Given $\varepsilon, T>0$ an infinite $(\varepsilon, T)$-chain is a pair of doubly infinite sequences

$$
\left\{\ldots, x_{-2}, x_{-1}, x_{0}, x_{1}, x_{2}, \ldots ; \ldots, t_{-2}, t_{-1}, t_{0}, t_{1}, t_{2}, \ldots\right\}
$$

such that $t_{i} \geqslant T$ and $d\left(x_{i} \cdot t_{i}, x_{i+1}\right)<\varepsilon$ for all $i(d$ denotes the distance in $M)$. Let $x_{0} * t$ denote the point on this chain $t$ units from $x_{0}$, i.e., if $t>0$ then

$$
x_{0} * t=x_{i} \cdot\left(t-\sum_{n=0}^{i-1} t_{n}\right) \text { where } \sum_{n=0}^{i-1} t_{n} \leqslant t<\sum_{n=0}^{i} t_{n},
$$

and if $t<0$ then

$$
x_{0} * t=x_{i} \cdot\left(t+\sum_{n=i}^{-1} t_{n}\right) \text { where }-\sum_{n=i}^{-1} t_{n} \leqslant t<-\sum_{n=i+1}^{-1} t_{n} .
$$

Here $\sum_{n=p}^{q} t_{n}=0$ if $q<p$.

Given $x, y \in M$ and $\varepsilon, T>0$, an $(\varepsilon, T)$-chain from $x$ to $y$ is a finite 
sequence of points and times, as above, with $x_{0}=x$ and $x_{n}=y$. A point $x$ is chain recurrent if for every $\varepsilon, T>0$ there is an $(\varepsilon, T)$-chain from $x$ to $x$. The set of chain recurrent points is called the chain recurrent set $R . R$ is a closed invariant set containing $\Omega$. A compact invariant subset $\Lambda$ of a compact metric space is chain recurrent if $\mathscr{R}\left(\left.f\right|_{\Lambda}\right)=\Lambda$. C. Conley [2] has shown that $R\left(\left.f\right|_{\mathscr{R}}\right)$ $=\Re$, i.e., the chain recurrent set is chain recurrent.

With certain hyperbolicity assumptions, it is possible to approximate infinite $(\varepsilon, T)$-chains with actual orbits. More precisely, an orbit $y \cdot \mathbf{R}$ is said to $\delta$-trace an infinite $(\varepsilon, T)$-chain $x_{0} * \mathbf{R}$ if there is an orientation preserving homeomorphism $g$ of $\mathbf{R}$ fixing the origin such that $d\left(x_{0} * t, y \cdot g(t)\right)<\delta$ for all $t \in \mathbf{R}$. We call $g$ a reparametrization of $y \cdot \mathbf{R}$. In [5] we show:

LEMMA 2.1. Let $\Lambda$ be a hyperbolic closed invariant set. Given $\delta>0$ there is an $\varepsilon>0$ so that every $(\varepsilon, 1)$-chain in $\Lambda$ can be $\delta$-traced by some $x \in M$. In addition the range of the reparametrization $g$ will be large if the total time of the $(\varepsilon, 1)$-chain is large.

LEMMA 2.2. Let $\Lambda$ be a hyperbolic closed invariant set. There exists $\delta>0$ so that for each $\varepsilon>0$ there is $S(\varepsilon)>0$ such that, if

(i) $x, y \in \Lambda$,

(ii) $g$ and $h$ are reparametrizations of $x \cdot \mathbf{R}$ and $y \cdot \mathbf{R}$, respectively,

(iii) $0 \in\left[t_{1}, t_{2}\right] \subset \mathbf{R}$,

(iv) $d(x \cdot g(t), y \cdot h(t))<\delta$ for all $t \in\left[t_{1}, t_{2}\right]$,

(v) $[-S(\varepsilon), S(\varepsilon)] \subset\left[h\left(t_{1}\right), h\left(t_{2}\right)\right] \cap\left[g\left(t_{1}\right), g\left(t_{2}\right)\right]$,

then $d(x, y \cdot s)<\varepsilon$ for some $s$ where $|s|<1$. Moreover, if $\left[t_{1}, t_{2}\right]=\mathbf{R}$ then $x=y \cdot s$.

Another result which follows from Lemmas 2.1 and 2.2 is

LemMa 2.3. Let $\Lambda$ be a hyperbolic closed invariant set. Given $\delta>0$ there is an $\varepsilon>0$ such that every periodic $(\varepsilon, 1)$-chain is $\delta$-traced by a periodic orbit.

Using the previous three lemmas we can prove [5].

THEOREM 2.4. $\Re$ is hyperbolic if and only if $f$ satisfies Axiom $\mathrm{A}$ and has no cycles. Also, in this case $\mathfrak{R}=\Omega$.

In [1] we analyze the relationship between hyperbolicity and quasi-hyperbolicity in the presence of chain recurrence.

THEOREM 2.5. Let $\Lambda$ be a compact chain recurrent subset of $M . \Lambda$ is hyperbolic if and only if $\Lambda$ is quasi-hyperbolic.

CoRollary 2.6. $\Re$ is quasi-hyperbolic if and only if $f$ satisfies Axiom $\mathrm{A}$ and has no cycles. 
3. Spectral Decomposition Theorem. Knowing that an invariant set has a finite number of connected components is often useful. In this section we use tracing and chain recurrence to show that certain invariant sets have a finite number of components. First we need two lemmas.

Definition. Let $\left\{C_{i}\right\}$ be a sequence of sets in $M$. Define $\mathcal{L}\left\{C_{i}\right\}=\{y \in$ $M: \exists\left\{y_{i}\right\}, y_{i} \in C_{i}$, which has $y$ as an accumulation point $\}$. Note that $\mathcal{L}\left\{C_{i}\right\}$ is closed.

LEMMA 3.1. Let $\left\{C_{i}\right\}$ be a sequence of connected sets in $M$. If there is a sequence $\left\{x_{i}\right\}, x_{i} \in C_{i}$, which converges, then $\mathcal{L}\left\{C_{i}\right\}$ is connected.

Proof. Let $x=\lim x_{i}$ and suppose $A, B$ is a separation of $\mathscr{L}\left\{C_{i}\right\}$ with $x \in A$. Choose $\left\{y_{i}\right\}, y_{i} \in C_{i}$, with accumulation point $y$ in $B$. Let $y=\lim _{k} y_{i_{k}}$ where $y_{i_{k}} \in C_{i_{k}}$. Since $\mathcal{L}\left\{C_{i}\right\}$ is closed, there are disjoint open sets $U, V$ in $M$ such that $A \subset U$ and $B \subset V$. For sufficiently large $k, x_{i_{k}} \in U$ and $y_{i_{k}} \in V$. Hence $C_{i_{k}} \cap U \neq \varnothing$ and $C_{i_{k}} \cap V \neq \varnothing$. Since $C_{i_{k}}$ is connected, $C_{i_{k}} \cap(M-U$ $\cup V) \neq \varnothing$ for $k$ large. Let $z_{i_{k}} \in C_{i_{k}} \cap(M-U \cup V)$ and $z$ be an accumulation point of $\left\{z_{i_{k}}\right\}$. Now $z \in \mathcal{L}\left\{C_{i}\right\}$ but $z$ is outside of $U \cup V \supset A \cup B=$ $\mathfrak{L}\left\{C_{i}\right\}$. This contradiction shows that $\mathfrak{L}\left\{C_{i}\right\}$ is connected.

Lemma 3.2. Let $\Lambda$ be a hyperbolic chain recurrent set. Suppose $\Lambda$ has a neighborhood $N$ such that $N-\Lambda$ contains no periodic orbits. Then each component of $\Lambda$ has a neighborhood $U$ such that no other component of $\Lambda$ is a subset of $U$.

Proof. Suppose not. Let $\Lambda_{1}$ be a component which does not have such a neighborhood. Let $\delta$ be as given in Lemma 2.2 and less than the distance from $\Lambda$ to $M-N$. By Lemmas 2.1 and 2.3 there is a $0<\varepsilon<\delta / 2$ such that every $(\varepsilon, 1)$-chain can be $\delta / 2$ traced and if the chain is periodic then the tracing orbit is periodic. Let $G$ be the $\varepsilon / 2$ neighborhood of $\Lambda_{1}$ and let $\Lambda_{2}$ be another component of $\Lambda$ contained in $G$. Choose $x \in \Lambda_{1}$ and $y \in \Lambda_{2}$ with $d(x, y)<\varepsilon / 2$. Let $\delta_{i}$ be a sequence of positive numbers decreasing to 0 . By Lemmas 2.1 and 2.3 there are $\varepsilon_{i}<\varepsilon / 2$ corresponding to these $\delta_{i}$. Pick two sequences $\left\{\alpha_{i}\right\}$ and $\left\{\beta_{i}\right\}$ of $\left(\varepsilon_{i}, 1\right)$-chains in $\Lambda$ where each $\alpha_{i}$ goes from $x$ to $x$ and each $\beta_{i}$ goes from $y$ to $y$. We construct a periodic infinite $\left(\varepsilon_{i}, 1\right)$-chain by repeating $\alpha_{i}$ in both time directions. By Lemma 2.3 this orbit is $\delta_{1}$-traced by a periodic orbit $a_{i}$. Doing the same procedure with $\beta_{i}$ gives periodic orbits $b_{i}$. Since there are sequences $\left\{x_{i}\right\}, x_{i} \in a_{i}$, and $\left\{y_{i}\right\}, y_{i} \in b_{i}$, with $x=\lim x_{i}$ and $y=\lim y_{i}, x \in \mathcal{L}\left\{a_{i}\right\}$ and $y \in \mathcal{L}\left\{b_{i}\right\}$.

Another way to produce a sequence of periodic infinite $(\varepsilon, 1)$-chains $\left\{\gamma_{i}\right\}$ is to let $\gamma_{i}$ be formed by doing $\alpha_{i} i$ times then $\beta_{i} i$ times and then repeating this in both time directions. Note this might not be an $\left(\varepsilon_{i}, 1\right)$-chain because the jump from $\alpha_{i}$ to $\beta_{i}$ is only guaranteed to be less than $\varepsilon$. Lemma 2.3 tells us that each $\gamma_{i}$ can be $\delta / 2$-traced by a periodic orbit $c_{i}$. Now $c_{i}$ and $a_{i} \delta$-trace 
each other when they are both tracing $\alpha_{i}$. As $i$ increases the time $a_{i}$ and $c_{i}$ spend tracing each other increases. By applying Lemma 2.2 we see that there are points on $a_{i}$ and $c_{i}$ which become arbitrarily close as $i$ increases. In fact there are points $z_{i}$ on $c_{i}$ so that $\lim d\left(z_{i}, x_{i}\right)=0$. It follows that $x=\lim z_{i}$ and so $x \in \mathcal{L}\left(c_{i}\right)$. The same argument using $b_{i}$ shows $y \in \mathcal{L}\left\{c_{i}\right\}$.

Since $\Lambda$ is closed and contains all nearby periodic orbits, $\mathscr{E}\left\{c_{i}\right\} \subset \Lambda$. Since $\mathcal{L}\left\{c_{i}\right\}$ is connected (Lemma 3.1) it must be in one component of $\Lambda$. But $\mathcal{L}\left\{c_{i}\right\}$ has points in both $\Lambda_{1}$ and $\Lambda_{2}$. This contradiction finishes the proof.

Theorem 3.3. Spectral Decomposition. Let $f$ be a $C^{1}$ flow on a compact connected manifold $M$. Suppose $\Lambda \subset M$ is quasi-hyperbolic and $\Lambda$ has $a$ neighborhood $N$ such that $N-\Lambda$ contains no periodic orbits. Then $\Lambda$ has a finite number of components. If, in addition, $\Lambda$ is chain recurrent then the periodic orbits are dense in $\Lambda$ and each component $\Lambda_{i}$ contains an $x$ such that $\alpha(x)=\omega(x)=\Lambda_{i}$.

Proof. Since the $\omega$-limit set of any point in $\Lambda$ is a subset of $\mathcal{R}\left(\left.f\right|_{\Lambda}\right)$, then every component of $\Lambda$ contains a component of $\mathcal{R}\left(\left.f\right|_{\Lambda}\right)$. Thus the number of components of $\Lambda$ is less than or equal to the number of components of $\Re\left(\left.f\right|_{\Lambda}\right)$.

To prove the first implication assume $\mathscr{R}\left(\left.f\right|_{\Lambda}\right)$ has an infinite number of components $\left\{\Lambda_{i}\right\}$. Let $x$ be an accumulation point of $\left\{x_{i}\right\}, x_{i} \in \Lambda_{i}$. Assume $x=\lim x_{i_{k}}$. Let $\Lambda^{\prime}$ be the component of $R\left(\left.f\right|_{\Lambda}\right)$ that contains $x$. Since $R\left(\left.f\right|_{\Lambda}\right)$ is quasi-hyperbolic and chain recurrent, Theorem 2.5 implies $R\left(\left.f\right|_{\Lambda}\right)$ is hyperbolic. All periodic points of $\Lambda$ are in $\mathcal{R}\left(\left.f\right|_{\Lambda}\right)$ so Lemma 3.2 gives a neighborhood $U$ of $\Lambda^{\prime}$ such that no other component of $\mathcal{R}\left(\left.f\right|_{\Lambda}\right)$ is a subset of $U$.

By Lemma 3.1, $\mathcal{L}\left\{\Lambda_{i_{k}}\right\}$ is a connected subset of $\mathcal{R}\left(\left.f\right|_{\Lambda}\right)$. Since each $\Lambda_{i_{k}}$ contains a point not in $U$, there is a point in $\mathcal{L}\left\{\Lambda_{i_{k}}\right\}$ not in $\Lambda^{\prime}$. But $x \in \mathcal{L}\left\{\Lambda_{i_{k}}\right\}$. Since $\mathcal{L}\left\{\Lambda_{i_{k}}\right\}$ is contained in one component we have a contradiction. Hence $\Lambda$ has a finite number of components.

Now assume $\Lambda$ is chain recurrent. Since periodic orbits close to $\Lambda$ are actually in $\Lambda$, Lemma 2.3 tells us that the periodic orbits are dense in $\Lambda$.

Consider a component $\Lambda_{1}$ of $\Lambda$. Since $\Lambda$ is chain recurrent, C. Conley [2] has shown that if $x, y \in \Lambda_{1}$ and $\varepsilon, T>0$ then there is an $(\varepsilon, T)$-chain from $x$ to $y$ in $\Lambda_{1}$. We now show that the positive orbit of each open subset $U$ of $\Lambda_{1}$ is dense in $\Lambda_{1}$. Let $x \in \Lambda_{1}$ and $\varepsilon_{1}>0$. Pick $y \in U$. Let $\varepsilon_{2}$ be the distance from $y$ to $\Lambda_{1}-U$ and $\delta=\min \left(\varepsilon_{1}, \varepsilon_{2}\right)$. There is an $\varepsilon_{3}>0$ such that each periodic $\left(\varepsilon_{3}, 1\right)$-chain can be $\delta$-traced with a periodic orbit. Construct a periodic $\left(\varepsilon_{3}, 1\right)$-chain containing $x$ and $y$. The corresponding $\delta$-tracing periodic orbit passes through $U$ and is within $\varepsilon_{3}$ of $x$. This shows that the positive as well as negative orbits of $U$ are dense in $\Lambda_{1}$. Now by a standard Baire Category 
argument [9] there is an $x \in \Lambda_{1}$ with $\alpha(x)=\omega(x)=\Lambda_{1}$. This completes the proof.

A closed invariant set is isolated if it is the maximal invariant set within some neighborhood of itself. Clearly an isolated invariant set $\Lambda$ has a neighborhood $N$ such that $N-\Lambda$ contains no periodic orbits. Thus we have

COROLlaRY 3.4. If $\Lambda$ is a quasi-hyperbolic isolated invariant set then $\Lambda$ has finitely many components.

Corollary 3.5. Smale's Spectral Decomposition Theorem. If $\Omega$ is Axiom A then $\Omega$ has a finite number of components and each component has a transitive orbit.

Proof. This follows from the fact that $\Omega=\mathrm{Cl} P$ which is a chain recurrent set.

We also get flow versions of results proved by $\mathrm{S}$. Newhouse [8] in the diffeomorphism case.

Corollary 3.6. If $L^{+}=\mathrm{Cl} \cup_{x \in M} \omega(x)($ or $\mathrm{Cl} P)$ is hyperbolic then $L^{+}=$ $\mathrm{Cl} P$ and has $a$ finite number of components; and each component has a transitive orbit.

Proof. To show $L^{+}$is chain recurrent and thus apply Theorem 3.3, we need the following:

LEMMA 3.7. For each $x \in M, \omega(x)$ is a chain recurrent set.

Proof. Let $y \in \omega(x)$ and take $\varepsilon, T>0$. We construct an $(\varepsilon, T)$-chain from $y$ to $y$ that stays in $\omega(x)$. There is a $0<\delta<\varepsilon / 2$ such that if $d(z, w)<\delta$ then $d(z \cdot t, w \cdot t)<\varepsilon / 2$ for $|t|<2 T$. There is an $S>0$ such that $x \cdot[S, \infty)$ is in the $\delta$-neighborhood of $\omega(x)$. There is a $T_{0}>S$ such that $d\left(x \cdot T_{0}\right.$, $y)<\delta$. So we start the $(\varepsilon, T)$-chain at $y$ and go for time $T$. Now $d(y \cdot T$, $\left.x \cdot\left(T_{0}+T\right)\right)<\varepsilon / 2$. Similarly $x \cdot\left(T_{0}+T\right)$ is in the $\delta$-neighborhood of $\omega(x)$. So pick $y_{1} \in \omega(x)$ within $\delta$ of $x \cdot\left(T_{0}+T\right)$ to be on the chain. Note that $d\left(y \cdot T, y_{1}\right)<\varepsilon$. Continuing in this manner produces an $(\varepsilon, T)$-chain that stays within $\varepsilon / 2$ of the orbit of $x$. There is a time $T_{1}>T_{0}+T$ such that $d\left(x \cdot T_{1}, y\right)<\varepsilon / 2$. Find the point $z$ on the $(\varepsilon, T)$-chain that corresponds with $x \cdot\left(T_{1}-T\right)$. Now $d\left(z \cdot T, x \cdot T_{1}\right)<\varepsilon / 2$ because it is less than $2 T$ along an orbit segment that started within $\delta$ of the orbit of $x$. Thus $d(z \cdot T, y)<\varepsilon$ and we have an $(\varepsilon, T)$-chain from $y$ to $y$.

4. Cycle points. In this section we discuss cycles without the assumption of Axiom A.

A point $x$ is a cycle point if $x$ is an element of a finite sequence of points $\left\{x=x_{0}, x_{1}, \ldots, x_{n}=x\right\}$ with $\omega\left(x_{i}\right) \cap \alpha\left(x_{i+1}\right) \neq \varnothing \forall 0<i<n-1$. Let $\mathcal{C}$ be the closure of the set of cycle points. The next lemma shows when Smale's 
no cycle property is equivalent to $\Omega$ containing all cycle points.

LEMMA 4.1. Let $f$ be an Axiom A flow. Then $\mathcal{C} \subset \Omega$ if and only if $f$ has no cycles.

Proof. To show necessity suppose $\Omega_{0}, \ldots, \Omega_{n}$ is a cycle. Then $x \in$ $W^{u}\left(\Omega_{0}\right) \cap W^{s}\left(\Omega_{1}\right)$ is a cycle point because each $\Omega_{i}$ contains an $x_{i}$ with $\alpha\left(x_{i}\right)=\omega\left(x_{i}\right)=\Omega_{i}$. Note $x \notin \Omega$ because $\Omega_{0}$ and $\Omega_{1}$ are closed and disjoint. Thus $\bigodot \not \subset \Omega$.

Let $x_{0} \in \mathcal{C}-\Omega$. Let $\left\{x_{0}, x_{1}, \ldots, x_{n}\right\}$ be a finite sequence with $x_{0}=x_{n}$ and $\omega\left(x_{i}\right) \cap \alpha\left(x_{i+1}\right) \neq \varnothing$. Let $\Omega_{i}$ be the component of $\Omega$ which contains $\omega\left(x_{i}\right)$. Note $\Omega_{0}=\Omega_{n}$. By an argument similar to the cloud lemma [10], $\alpha\left(x_{0}\right)$ and $\omega\left(x_{0}\right)$ are in different components of $\Omega$. Hence $\Omega_{0} \not \subset \Omega_{n-1}$. Now eliminating the repetitions in $\Omega_{0}, \Omega_{1}, \ldots, \Omega_{n}$ gives a cycle.

If $x \in \mathcal{C}$ then a corresponding sequence of cycle points containing $x$ can be used to produce $(\varepsilon, T)$-chains from $x$ to $x$. Hence,

LEMMA 4.2. $\mathrm{Cl} P \subset \mathcal{C} \subset \Re$. In fact $\mathcal{C}$ is a chain recurrent set.

Corollary 4.3. If $\Re$ is hyperbolic then $\mathrm{Cl} P=\Omega=\mathcal{C}=\Re$.

Proof. Use Theorem 2.4 and Lemma 4.2.

EXAMPLe 4.4. This example shows that $C$ is not always equal to $R$. Let $f$ be the flow on a rectangle as illustrated in Figure 1. Every interior point moves straight down and the boundary points are fixed. Every point is chain recurrent but only the fixed points are cycle points.

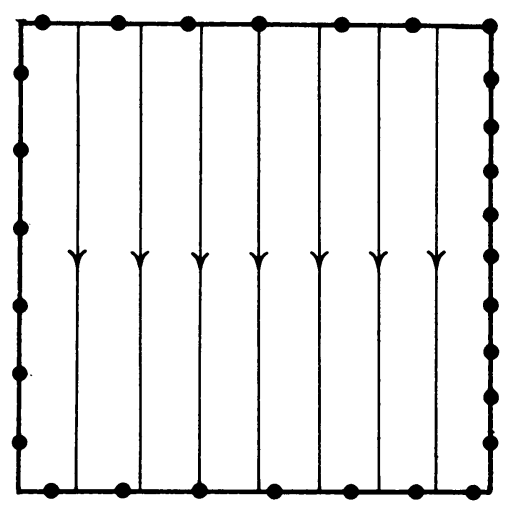

FIGURE 1

Another important chain recurrent set is a minimal set. A set $\Lambda$ is minimal if it is closed, invariant and has no nonempty proper subset which is closed and invariant. For each $x \in \Lambda, \alpha(x)=\omega(x)=\Lambda$. Hence, by Lemma 3.7, $\Lambda$ is a chain recurrent set. Let $\Re$ be the closure of the union of all minimal sets. 
LEMMA 4.5. $\mathrm{Cl} P \subset \Re \subset \mathcal{C}$. In fact $\Re$ is chain recurrent.

Proof. Let $x$ be in a minimal set. Since $\alpha(x)=\omega(x),\{x, x\}$ is a legitimate sequence of cycle points.

COROLlaRY 4.6. If $\Re$ is quasi-hyperbolic then $\Re$ has finitely many components; and each component is the closure of its periodic orbits and has a transitive orbit.

Proof. This follows from Theorem 3.3 and the fact that $\mathfrak{R}$ is a chain recurrent set.

5. When are chain recurrent points cycle points? The intent of this section is to prove Theorem $\mathrm{C}$. We will show that every chain recurrent point is a cycle point when $\mathfrak{T}$ is hyperbolic. The argument involves several technical lemmas.

Let $\Lambda$ be a closed set and $\delta>0$. Define

$$
N_{\delta}(\Lambda) \equiv\{x: d(x, \Lambda)<\delta\} .
$$

Lemma 5.1. Let $f$ be a flow on $M$. Then for each $\delta>0$, there is $T>0$ such that for all $x \in M-N_{\delta}(\mathfrak{T})$

$$
x \cdot[0, T] \cap N_{\delta}(\Re) \neq \varnothing \text {, and } x \cdot[-T, 0] \cap N_{\delta}(\mathscr{T}) \neq \varnothing .
$$

Proof. Given $x \in M-N_{\delta}(\Re)$ there is a $T_{x}>0$ such that $x \cdot T_{x} \in$ $N_{\delta}(\Re)$ because $\omega(x)$ contains a minimal set. By continuity this is true for a neighborhood of $x$. Take a finite subcover of $M-N_{\delta}(\mathfrak{T})$. For the maximum $T$ of these $T_{x}$ 's we have

$$
x \cdot[0, T] \cap N_{\delta}(\mathfrak{T}) \neq \varnothing \quad \text { for all } x \in M-N_{\delta}(\mathfrak{T}) .
$$

We can find a similar $T$ for negative times. Let $T$ be the maximum of these two times.

LEMMA 5.2. Given $\delta>0$ and $x \in R$ then for each $\varepsilon, T>0$ there is $a$ periodic $(\varepsilon, T)$-chain which contains $x \cdot[-T, T]$ on a segment and all the jumping occurs in $N_{\delta}(\mathfrak{N})$.

Proof. Given $\delta, \varepsilon, T>0$ and $x \in \Re$, we construct the required $(\varepsilon, T)-$ chain. Let $T_{1}>0$ be the time corresponding to $\delta / 2$ in Lemma 5.1. Choose $\delta_{1}$ such that if $d(y, z)<\delta_{1}$ then $d(y \cdot t, z \cdot t)<\min \{\delta / 2, \varepsilon\}$ for $|t|<T_{1}+T$. Let $\varepsilon_{1}<\min \left\{\delta_{1}, \delta / 2, \varepsilon\right\}$ and $T_{2} \equiv 2 T_{1}+2 T$. Take an $\left(\varepsilon_{1}, T_{2}\right)$-chain $\{x=$ $\left.x_{0}, x_{1}, x_{2}, \ldots, x_{n}=x\right\}$. Let $y$ be the endpoint of the first segment of this $\left(\varepsilon_{1}, T_{2}\right)$-chain. Then there is a $t_{1} \in\left[0, T_{1}\right]$ such that $y \cdot t_{1} \in N_{\delta / 2}(\Re)$. Since $d\left(y, x_{1}\right)<\varepsilon_{1}, d\left(y \cdot t_{1}, x_{1} \cdot t_{1}\right)<\min \{\delta / 2, \varepsilon\}$. Thus an $\varepsilon$ jump can be made from the orbit of $x$ to the orbit of $x_{1}$ within $\delta$ of $\Re$. Note that there is at least 
$T_{1}+2 T$ time left on the segment that started at $x_{1}$. Consider the end of this segment and repeat the procedure.

To finish the $(\varepsilon, T)$-chain we need to modify the last segment. Note there is a $T \leqslant t \leqslant T+T_{1}$ such that $x \cdot(-t) \in N_{\delta / 2}(\mathfrak{T})$. If $y_{n}$ is the endpoint of the last segment then $d\left(y_{n} \cdot(-t), x \cdot(-t)\right)<\min \{\delta / 2, \varepsilon\}$. Jumping from $y_{n} \cdot$ $(-t)$ to $x \cdot(-t)$ shortens the last segment by a total of at most $2 T_{1}+T$ leaving at least $T$ time units. Since $y_{n} \cdot(-t)$ is within $\delta$ of $\Re$ we have constructed a periodic $(\varepsilon, T)$-chain with the desired properties.

LEMMA 5.3. Let $\Re$ be quasi-hyperbolic and $\left\{\varepsilon_{i}\right\}$ be a sequence strictly decreasing to 0 . Suppose $\mathfrak{K}_{1}$ and $\mathfrak{K}_{2}$ are components of $\mathfrak{R}_{\text {and }}\left\{s_{i}\right\}$ is a sequence of orbit segments so that for each $i$ the initial point of $s_{i}$ is within $\varepsilon_{i}$ of $\mathfrak{T}_{1}$ and final point of $s_{i}$ is within $\varepsilon_{i}$ of $\mathfrak{T}_{2}$. Then there is a finite sequence of points $\left\{x_{1}, \ldots, x_{n}\right\}$ such that $\alpha\left(x_{1}\right) \cap \mathfrak{T}_{1} \neq \varnothing, \omega\left(x_{n}\right) \cap \mathfrak{T}_{2} \neq \varnothing$ and $\alpha\left(x_{i+1}\right) \cap \omega\left(x_{i}\right) \neq \varnothing$ for $1 \leqslant i \leqslant n-1$.

PRoof. Since $\Re$ is quasi-hyperbolic, Corollary 4.5 guarantees that $\mathscr{N}$ has finitely many components. Without loss of generality we can assume $\varepsilon_{1}$ is smaller than half the distance between the closest two components. By Lemma 5.1, for each $i$ there is a $T_{i}>0$ such that $x \cdot\left[0, T_{i}\right]$ and $x \cdot\left[-T_{i}, 0\right]$ intersect $N_{e_{i}}(\Re)$ for all $x \in M$.

We construct two finite sequences of points on each $s_{i}$. Let $z_{i}^{i}$ be the last point on the segment $s_{i}$ that is $\varepsilon_{i}$ from $\Re_{1}$. Let $w_{i}^{i}$ be the next point on $s_{i}$ beyond $z_{i}^{i}$ that is $\varepsilon_{i}$ from $\Re$. These points exist and are distinct because $\varepsilon_{1}$ is less than half the distance between the closest two components. Let $z_{i}^{i-1}$ be the last point on the segment from $z_{i}^{i}$ to $w_{i}^{i}$ that is within $\varepsilon_{i-1}$ of $\mathfrak{T}_{1}$. Choose $w_{i}^{i-1}$ to be the next point beyond $z_{i}^{i-1}$ that is within $\varepsilon_{i-1}$ of $\Re$. Inductively, for each $1 \leqslant j \leqslant i$, we define $z_{i}^{j}$ to be the last point on the segment from $z_{i}^{j+1}$ to $w_{i}^{j+1}$ that is within $\varepsilon_{j}$ of $\Re_{1}$. Likewise $w_{i}^{j}$ is the next point beyond $z_{i}^{j}$ that is within $\varepsilon_{j}$ of $\Re$.

Let $y_{1}$ be a limit point of $\left\{z_{i}^{1}\right\}$.

Claim. $\alpha\left(y_{1}\right) \cap \mathbb{R}_{1} \neq \varnothing$ and $\omega\left(y_{1}\right) \cap \Re-\Re_{1} \neq \varnothing$.

Proof of Claim. We show the positive orbit of $y_{1}$ gets arbitrarily close to $\Re-\Re_{1}$ and thus $\omega\left(y_{1}\right) \cap \Re-\Re_{1} \neq \varnothing$. The same argument works in negative time to show $\alpha\left(y_{1}\right) \cap \mathfrak{R}_{1} \neq \varnothing$.

Let $\varepsilon>0$ and choose an $i$ such that $\varepsilon_{i}<\varepsilon / 2$. There is a $\delta>0$ such that if $d(x, y)<\delta$ then $d(x \cdot t, y \cdot t)<\varepsilon / 2$ for $|t|<T_{i}$. Let $z_{j}^{1}$ be a point in the sequence $\left\{z_{k}^{1}\right\}$ that is within $\delta$ of $y_{1}$ where $j>i$. By construction, $z_{j}^{1}$ is between $z_{j}^{i}$ and $w_{j}^{i}$ on $s_{j}$. Since the time from $z_{j}^{1}$ and $w_{j}^{i}$ is less than $T_{i}$, there is a time $0<t<T_{i}$ so that $z_{j}^{1} \cdot t$ is within $\varepsilon_{i}<\varepsilon / 2$ of $\mathscr{T}-\mathfrak{T}_{1}$. Since $d\left(z_{j}^{1} \cdot t\right.$, $\left.y_{1} \cdot t\right)<\varepsilon / 2$, the triangle inequality implies that $y_{1} \cdot t$ is within $\varepsilon$ of $\mathfrak{N}-$ $\mathfrak{T}_{1}$. This completes the proof of the claim.

Note that the one-point sequence $x_{1}=y_{1}$ provides a sequence which 
connects $\Re_{1}$ with any component of $\Re$ meeting $\omega\left(y_{1}\right)$, as desired in the conclusions of the theorem. If $\omega\left(y_{1}\right)$ intersects $\mathfrak{T}_{2}$, we are done. If not let $\Re^{1}$ denote $\Re_{1}$ union the components of $\Re$ which meet $\omega\left(y_{1}\right)$. Apply the previous argument to $\mathscr{T}^{1}$, in place of $\mathscr{T}_{1}$, and $\Re_{2}$. This gives a $y_{2}$ with the properties that $\alpha\left(y_{2}\right) \cap \mathscr{T}^{1} \neq \varnothing$ and $\omega\left(y_{2}\right) \cap \Re^{\prime}-\Re^{1} \neq \varnothing$. We can now construct a sequence as in the theorem connecting $\mathfrak{T}_{1}$ with any component that meets $\omega\left(y_{2}\right)$. We do this by picking a component of $\Re^{1}$ that meets $\alpha\left(y_{2}\right)$ and a transitive orbit in this component. Since $\mathfrak{T}_{1}$ can be connected to this component by an appropriate sequence, adding this transitive orbit and the orbit of $y_{2}$, we get an appropriate sequence connecting $\mathfrak{T}_{1}$ with any component meeting $\omega\left(y_{2}\right)$. If $\omega\left(y_{2}\right)$ meets $\mathfrak{K}_{2}$ we are done. If not, we continue this procedure until we exhaust the finite components of $\mathfrak{T}$. This completes the proof.

A modification of Example 4.4 gives an example where $\Re$ has an infinite number of components. Change one of the sides from being fixed to having a Cantor set of fixed points with the flow moving down in between them. Then each of the fixed points on this side is a component of $\mathfrak{T}$. Each pair of these fixed points can be connected as in the hypothesis of Lemma 5.3 using orbits in the interior, but the conclusion fails to hold.

With these lemmas we can prove the main theorem of this section.

THEOREM 5.4. If $\Re$ is hyperbolic, then $\Re=\mathcal{C}$.

Proof. Let $x \in R$. We will construct a sequence of cycle points containing $x$.

By Corollary 4.6, $\Re$ has a finite number of components. Choose a sequence $\left\{\delta_{i}\right\}$ strictly decreasing to 0 , with $\delta_{1}$ less than one fourth the minimum distance between components of $\Re$. Take $\varepsilon_{i}=\delta_{i}$ and $T_{i}>0$ such that $x \cdot t$ and $x \cdot(-t)$ are within $\delta_{i}$ of $\omega(x)$ and $\alpha(x)$, respectively, for $t \geqslant T_{i}$. Lemma 5.2 gives a sequence $\left\{\Gamma_{i}\right\}$ of periodic $\left(\varepsilon_{i}, T_{i}\right)$-chains with all the jumping in $N_{\delta_{i}}(\mathfrak{T})$. For each jump on each $\Gamma_{i}$ there is a unique component of $\mathfrak{N}$ so that the jumping is within $\delta_{i}$ of that component. Thus each $\Gamma_{i}$ produces a finite sequence of components of $\mathfrak{N}$ where the first intersects $\omega(x)$ and the last intersects $\alpha(x)$.

If a component appears twice in this sequence delete the components between the two appearances and delete one of the repeated components. Continue this procedure until all the components in the sequence are distinct. Therefore for each $i$ we have a finite sequence of components in which each term is distinct. The first component intersects $\omega(x)$ and the last component intersects $\alpha(x)$. Also for each pair of adjacent components there is an orbit segment which starts within $\delta_{i}$ of the first component and ends within $\delta_{i}$ of the second component.

Since $\Re$ has finitely many components, there are only a finite number of 
distinct finite sequences of components of $\mathfrak{N}$ which have no terms repeated. The above construction produced infinitely many such sequences. Hence one sequence $\left\{\Re_{1}, \ldots, \Re_{k}\right\}$ was the same for infinitely many $i$ 's. Take $\mathfrak{T}_{j}$ and $\mathfrak{N}_{j+1}$ for $1 \leqslant j \leqslant k-1$. By our construction there is a sequence of orbit segments $s_{i}$ starting closer and closer to $\mathfrak{T}_{j}$ and ending closer and closer to $\mathfrak{T}_{j+1}$.

Applying Lemma 5.3 gives a finite sequence of points $\left\{x_{1}^{j}, \ldots, x_{n(j)}^{j}\right\}$ such that $\alpha\left(x_{1}^{j}\right) \cap \Re_{j} \neq \varnothing, \omega\left(x_{n}\right) \cap \Re_{j+1} \neq \varnothing$ and $\alpha\left(x_{i+1}^{j}\right) \cap \omega\left(x_{1}^{j}\right) \neq \varnothing$ for $1 \leqslant i$ $\leqslant n-1$. Let $y_{j}$ be the orbit in $\mathfrak{N}_{j}$ with $\alpha\left(y_{j}\right)=\omega\left(y_{j}\right)=\mathfrak{N}_{j}$. Now $x, y_{1}, x_{1}^{1}$, $x_{2}^{1}, \ldots, x_{n}^{1}, y_{2}, x_{1}^{2}, x_{2}^{2}, \ldots, y_{k}$ is an appropriate sequence of cycle points. Thus $x \in \mathrm{C}$.

Theorem 5.4 leads to a result which gives an equivalent condition for Axiom A plus no cycles. Other mathematicians [7], [8] have found equivalent conditions usually in the diffeomorphism case. Our result gives flow versions of these as corollaries.

THEOREM 5.5. Axiom A plus no cycles is equivalent to the existence of a quasi-hyperbolic compact invariant set $\Lambda$ which contains $\mathcal{C}$.

Proof. Given Axiom $A$ and no cycles Theorem 2.4 gives that $R$ is hyperbolic and Lemma 4.2 gives $\mathcal{C} \mathfrak{R}$. Thus $R$ will serve as a $\Lambda$.

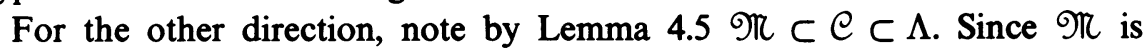
quasi-hyperbolic, Theorem 5.4 implies that $\Re=\bigodot$. Therefore Corollary 2.6 implies Axiom A plus no cycles.

Corollary 5.6 (Newhouse [8]). Axiom A plus no cycles is equivalent to $L^{+}$ being hyperbolic and containing $\mathcal{C}$.

Corollary 5.7 (MAÑE [7]). If $M$ is quasi-hyperbolic then the flow is Axiom A plus no cycles.

TheOREM 5.8. Axiom A plus no cycles $\Leftrightarrow \Omega$ being hyperbolic and containing $\mathrm{C} \Leftrightarrow \Omega$ being quasi-hyperbolic and containing $\mathrm{C}$.

Theorem 5.8 is important in light of an example recently announced by A. Dankner [4]. He has an example of a diffeomorphism with $\Omega$ hyperbolic but not Axiom A. Also, this theorem allows the hyperbolicity to be weakened.

TheOREM 5.9. $C$ being quasi-hyperbolic is equivalent to Axiom A plus no cycles.

Theorem 5.9 reduces Axiom A plus no cycles to locating a geometric set $\mathcal{C}$ and verifying, for $\mathcal{C}$, a condition weaker than hyperbolicity. In applications, one might approach the problem of finding $\mathcal{C}$ by first finding $\mathfrak{N}$. After verifying $\Re$ is quasi-hyperbolic, appeal to the Spectral Decomposition Theorem to show $\Re$ has finitely many components. If there are no cycle points 
connecting components of $\mathfrak{N}$ then we have $\mathcal{C} \subset \mathfrak{T}$ and hence Axiom A plus no cycles. If there is a cycle point connecting components of $\mathfrak{T}$ then $\Re \neq C$ and we do not have Axiom A plus no cycles.

Corollary 5.10. Axiom A plus no cycles $\Rightarrow \mathrm{Cl} P=\mathfrak{R}=\Omega=\mathcal{C}=\Re$.

Concerning stability, Corollary 5.10 shows that any condition that implies Axiom A plus no cycles gives the stability of each of the sets in this corollary.

\section{REFERENCES}

1. R. Churchill, J. Franke and J. Selgrade, A geometric criterion for hyperbolicity of flows, Proc. Amer. Math. Soc. 62 (1977), 137-143.

2. C. Conley, The gradient structure of a flow. I, IBM Research, RC 3932 (\# 17806), Yorktown Heights, New York, 1972.

3. Hyperbolic sets and shift automorphisms, Dynamical System Theory and Application, Springer-Verlag, New York, 1975, pp. 539-549.

4. A. Dankner, On Smale's Axiom A dynamical systems, Ann. of Math. 107 (1978).

5. J. Franke and J. Selgrade, Hyperbolicity and chain recurrence, J. Differential Equations 26 (1977), 27-36.

6. Abstract $\omega$-limit sets, chain recurrent sets, and basic sets for flows, Proc. Amer. Math. Soc. 60 (1976), 309-316.

7. R. Mañé, Quasi-Anosov diffeomorphisms and hyperbolic manifolds, Trans. Amer. Math. Soc. 229 (1977), 351-370.

8. S. Newhouse, Hyperbolic limit sets, Trans. Amer. Math. Soc. 167 (1972), 125-150.

9. Z. Nitecki, Differentiable dynamics, M.I.T. Press, Cambridge, Mass., 1971.

10. C. Pugh and M. Shub, The S-stability theorem for flows, Inventiones Math. 11 (1970), 150-158.

11. S. Smale, Differentiable dynamical systems, Bull. Amer. Math. Soc. 73 (1967), 747-817.

Department of Mathematics, North Carolina State University, Raleigh, North Carolina 27607 